\title{
Parálisis facial por angioma cavernoso del peñasco. Caso clínico
}

\author{
J.M. González-Darder y J.V. Pesudo-Martínez
}

Servicio de Neurocirugía. Hospital General de Castellón. Castellón

\section{Resumen}

La parálisis facial secundaria a compresión tumoral extrínseca del nervio facial en el ganglio geniculado es una eventualidad rara. Se presenta el caso de un paciente con angioma cavernoso del hueso temporal y clínica de parálisis facial periférica de evolución tórpida (House-Brackmann grado IV), acompañada de ojo seco y ausencia de reflejo estapedial. En el estudio de imagen rutinario con tomografía computarizada y resonancia magnética no se identificaron lesiones, pero las mismas técnicas realizadas tras la sospecha clínica identificaron una lesión menor de $1 \mathrm{~cm}$ con todas las características radiológicas de un angioma cavernoso. La lesión fue abordada y extirpada microquirúrgicamente a través de una vía de fosa media extradural con evolución favorable de los déficits neurológicos preoperatorios (House-Brackmann grado II).

PALABRAS CLAVE: Parálisis facial. Nervio facial. Tumor de peñasco. Angioma cavernoso óseo.

Facial nerve palsy due to cavernous angioma of the petrous bone. Case report

\section{Summary}

The facial nerve palsy due to extrinsic tumoral compression of the facial nerve at the geniculate ganglium is very rare. We present the case of a patient with a temporal bone cavernoma and symptoms of a torpid peripheral facial House-Brackmann grade IV nerve palsy with dry eye and loss of stapedial reflex. The routine computed tomographic and magnetic resonance imaging studies showed no abnormalities, but the same imaging techniques done after the clinical suspiction identified a less that $1 \mathrm{~cm}$ lesion that was compatible with an osseous cavernous angioma. The lesion was approached and removed through a microsurgical

Recibido: 3-01-06. Aceptado: 10-03-06 middle fossa extradural approach with a good postoperative recovery (House-Brackmann grade II).

KEY WORDS: Facial nerve palsy. Facial nerve. Petrous bone tumour. Osseous cavernous angioma.

\section{Introducción}

La parálisis facial secundaria a la compresión extrínseca del nervio facial por tumores del peñasco a nivel del ganglio geniculado es una eventualidad muy poco frecuente, pero constituye una entidad clínica muy recortada y con potencial resolución mediante descompresión microquirúrgica. En el presente trabajo presentamos un caso de parálisis facial de evolución tórpida debida a la compresión del nervio facial a nivel del ganglio geniculado por un angioma cavernoso del peñasco y resuelto mediante tratamiento microquirúrgico.

\section{Caso Clínico}

Se trata de un hombre de 34 años sin antecedentes de interés, que comienza ocho meses antes de la cirugía con una parálisis facial periférica derecha de evolución tórpida, tratada con diversas tandas de corticoides y complejos vitamínicos. Dada la mala evolución fue estudiada por diversos especialistas en otología y neurología. Los estudios de tomografía axial computarizada (TAC) y resonancia magnética (RNM) fueron considerados normales. Una nueva RNM, esta vez con contraste, permitió identificar una pequeña lesión captante de contraste en el techo del peñasco derecho y un estudio con cortes finos de TAC demostró una lesión intraósea que insuflaba la cortical y presentaba calcificaciones en su interior, sugestiva de angioma cavernoso (Fig.1). Remitido para tratamiento quirúrgico el paciente fue re-evaluado desde el punto de vista neurológico y otológico. Se determinó una parálisis facial periférica derecha grado IV de House y Brackmann (desviación de comisura bucal y de descenso palpebral, con cierre completo de ojo y boca) con sensación de ojo seco pero con test de Schirmer simétrico y reflejo estape- 


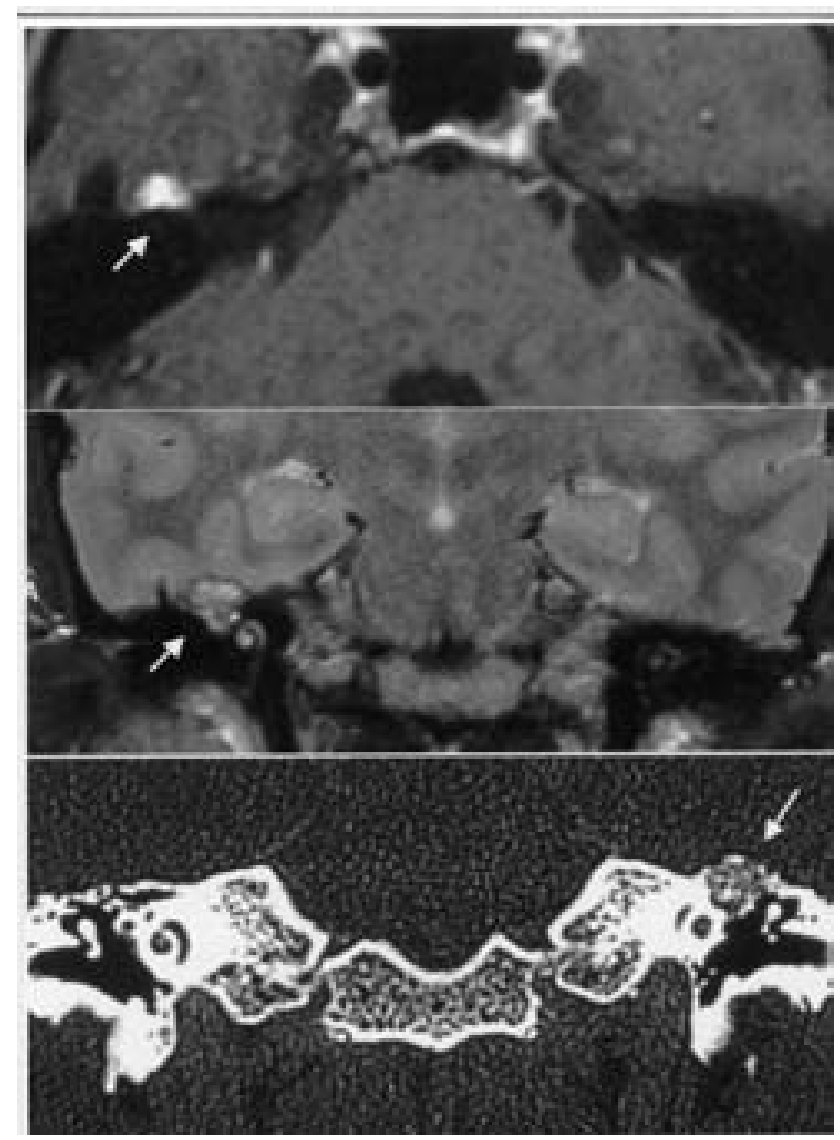

Figura 1. Imagen coronal de resonancia magnética mostrando la lesión en el techo del peñasco derecho (arriba) y la intensa captación de gadolinio de la misma en una imagen axial (centro). Imagen de tomografia computarizada en corte coronales mostrando las características típicas de la lesión y sus relaciones con el oído interno y cadena de huesecillos (abajo).

dial homolateral abolido. El paciente fue intervenido bajo anestesia general a través de un abordaje extradural de fosa media y se encontró en el techo del peñasco una lesión de aspecto típico de angioma cavernoso óseo, que fue fresada hasta descomprimir el nervio facial a nivel del ganglio geniculado y entrar en el oído medio exponiendo la cadena osicular. La evolución postoperatoria fue favorable, con sensación de ocupación del oído durante algunas semanas y mejoría de la parálisis facial (grado II de House y Brackmann: asimetría de surcos o pliegues dérmicos a la gesticulación) a los 10 meses. El estudio anatomopatológico confirmó el diagnóstico de angioma cavernoso óseo.

\section{Discusión}

Una parálisis facial periférica de evolución tórpida o acompañada de síntomas atípicos debe poner en guardia
2007; 18: 44--46

acerca de una posible etiología orgánica, especialmente sobre la existencia de un tumor que involucre el nervio facial en algún punto de su complejo trayecto anatómico, originado en el propio nervio, en el hueso temporal o en estructuras vecinas intracraneales o incluso extracraneales que invaden secundariamente el hueso y afectan de esa forma el nervio.

A pesar de su pequeño tamaño, las técnicas de imagen, en especial la TAC, demostraron en nuestro caso que nos encontrábamos ante una lesión intrapetrosa, con insuflación y erosión de la cortical acompañada de un moteado calcificado intralesional, bien delimitada y acompañada de intenso realce en la RNM tras administración de contraste. Todas éstas son características del angioma cavernoso intraóseo, que supone el $0.7 \%$ de los tumores del peñasco y que pueden localizarse en el conducto auditivo interno, ganglio geniculado e incluso conducto auditivo externo $^{1,4,5,7}$. La sintomatología típica del cavernoma del ganglio geniculado es la parálisis facial de evolución tórpida, aunque se ha descrito un caso de espasmo hemifacial secundario a cavernoma ${ }^{1}$. Al contrario de lo que ocurre con el neurinoma del facial, una característica del hemangioma es que produce clínica con un tamaño muy pequeño, habitualmente inferior a $1 \mathrm{~cm}$, lo que hace que se pierda en exploraciones rutinarias de TAC o RNM, tal y como ocurrió inicialmente en nuestro paciente. Ante una lesión tan pequeña y una clínica tan florida hay que considerar la fisiopatología de los síntomas para poder correlacionar la lesión, su topografía y los hallazgos ${ }^{3,8,9}$. El nervio facial vehicula diferentes tipos de fibras. Las fibras motoras recorren todo su trayecto intrapetroso saliendo por el agujero estilomastoideo, excepto las que se desprenden al inicio de la porción descendente del facial para inervar el músculo del estribo. La función de este músculo se explora mediante el reflejo estapedial, cuya abolición indica una lesión del facial proximal a la salida del nervio del músculo del estribo. Las fibras del parasimpático lacrimal se desprenden del facial en el ganglio geniculado formando parte del nervio petroso superficial mayor, para llegar a la glándula lacrimal a través de la primera rama del trigémino. La función lacrimal se explora mediante el clásico test de Schirmer. El parasimático salival y las fibras sensoriales gustativas se desprenden del facial a unos 2-3mm del agujero estilomastoideo formando la cuerda del tímpano, para llegar a sus destinos a través de la tercera rama del trigémino. Hay también tests específicamente destinados a estudiar la secreción salival y la función gustativa. Finalmente, el nervio facial también posee una zona cutánea de inervación a nivel del conducto auditivo externo, llamada zona de Ramsay Hunt, recogiendo estas fibras a través de un ramo que se incorpora al nervio unos milímetros por fuera del agujero estilomastoideo ${ }^{10}$. La lesión completa del nervio facial a nivel del ganglio geniculado y antes de la 
salida del nervio del estribo produce una parálisis motora acompañada de hiperacusia y afectación del reflejo estapedial por parálisis del músculo del estribo, trastorno de la secreción lacrimal y salival y de la funciones sensitivas. En nuestro paciente se objetivaba la parálisis, abolición del reflejo estapedial y un ojo seco, no pudiendo ser constatadas las otras eventuales afectaciones probablemente por tratarse de una lesión incompleta.

Para hacer un adecuado planteamiento terapéutico es muy importante determinar si estamos ante una lesión extrínseca del nervio facial. En cualquier caso, el manejo de una lesión focal que afecta de forma incompleta al nervio facial exige preservar al menos la función del nervio existente. Los diferentes casos publicados indican que la resección radical de un neurinoma del facial va seguida casi indefectiblemente de un deterioro clínico ${ }^{6,11,12}$. Por ello, el diagnóstico de un tumor intrínseco del facial con función motora aceptable plantea la duda de una actuación quirúrgica que puede llevar consigo el sacrificio del nervio y la necesidad de una reconstrucción que sólo asegura una recuperación parcial del mismo y, en todo caso, a largo plazo y no en todos los casos. Sin embargo, en caso de una lesión extrínseca, como el angioma cavernoso, la problemática es diferente ya que puede ser resecada a través de una vía de abordaje adecuada descomprimiendo eficazmente el nervio.

La vía de acceso a una lesión de esta topografía es el abordaje extradural de fosa media, utilizado clásicamente por los otólogos como vía de acceso a la patología del conducto auditivo interno ${ }^{2}$. El ganglio geniculado se encuentra a una media de $1.7 \mathrm{~cm}$ de la tabla interna estando tanto el ganglio como el trayecto inicial del nervio petroso mayor habitualmente cubiertos por una delgada capa de hueso. Para encontrar el ganglio geniculado se puede identificar el nervio petroso en su trayecto extraóseo y fresar su cubierta ósea retrógradamente hasta llegar al ganglio. También puede encontrarse en el ángulo que forman el trayecto del nervio petroso y el canal semicircular superior que se reconoce en la superficie petrosa como la eminencia arcuata9 Hallado el ganglio geniculado, fresando medialmente sobre una línea que forme $60^{\circ}$ con el nervio petroso o $30^{\circ}$ con la eminencia arcuata se accede al conducto auditivo interno; si se fresa lateralmente se accede a la cadena de huesecillos; si se fresa hacia detrás se llega al hueso compacto de los canales semicirculares; y, finalmente, si se fresa hacia delante siguiendo el trayecto de los nervios petrosos, se descubre la carótida intrapetrosa. En nuestro caso, la lesión se hizo evidente en el momento de llegar a la misma por vía extradural y el fresado y exéresis de la misma permitió exponer los elementos anatómicos referidos mas arriba.

En resumen, el cavernoma petroso del ganglio geni- culado produce una parálisis facial de evolución tórpida y puede diagnosticarse con técnicas de imagen. Dado que se trata de una lesión benigna, extraneural y que suele producir clínica cuando su tamaño es pequeño su tratamiento quirúrgico a través de un abordaje extradural de fosa media es curativo si el diagnostico es precoz.

\section{Bibliografía}

1. Asaoka, K., Sawamura, Y., Tada, M., Abe, H.: Hemifacial spasm caused by a hemangioma at the geniculate ganglion: case report. Neurosurgery 1997; 41: 1195-1197.

2. Brackmann, D.E., Hitselberger, W.E., Beneke, J.E., House, W.F.: Acoustic neuromas: middle fossa and translabyrinthine removal. En Rand, R.W.: Microneurosurgery. CV Mosby Co, St. Louis 1985; pp 311-334.

3. De Jong, R.N.: The Neurologic Examination. Harper\&Row, New York 1979.

4. Escada, P., Capucho, C., Silva, J.M., Ruah, C.B., Vital, J.P., Penha, R.S.: Cavernous haemangioma of the facial nerve. J. Laryngol. Otol. 1997; 111: 858-861.

5. Ginsberg, L.E., DeMonte, F.: Case 16: Facial nerve schwannoma with middle cranial fossa involvement. Radiology 1999; 213: 364-368.

6. González-García, J.A., Arenas-Britez, O., Gil-Carrasco, R., Lendoiro-Otero, C., Rodriguez-Paramás, A., Scola-Yurrita, B.: Tumores del ganglio geniculado. Manejo terapéutico y reconstructivo. Acta Otorrinolaringol. Esp. 2004; 5: 206-211.

7. Piccirillo, E., Agarwal, M., Rdhit, H., Khrais, T., Sanna, M.: Management of temporal bone hemangiomas. Ann. Otol. Rhinol. Laryngol 2004; 113: 431-437.

8. Testut, L., Latarjet, A.: Tratado de Anatomía Humana. Salvat, Barcelona 1969; vol. 3; pp 126-146.

9. Rothon, A.L.: The temporal bone and transtemporal approaches. Neurosurgery 2000; 47: S211-S265.

10. Samii, M., Jannetta, P.J.: The Cranial Nerves. SpringerVerlag, Berlin 1981; pp 178-198.

11. Sherman, J.D., Dagnew, E., Pertsak, M.L., van Loveren, H.R., Tew, J.M.: Facial nerve neuromas: report of 10 cases and review of the literature. Neurosurgery 2002; 50: 450-456.

12. Symon, L., Cheesman, A.D., Kawauchi, M., Bordi, L.: Neuromas of the facial nerve: a report of 12 cases. Br. J. Neurosurg 1993; 7: 13-22.

González-Darder, J.M.; Pesudo-Martínez, J.V.: Parálisis facial por angioma cavernoso del peñasco. Caso clínico. Neurocirugía 2007; 18: 44-46.

Dirección postal: Dr. José M. González-Darder. Servicio de Neurocirugía. Hospital General. Avda. Benicassim, s/n. 12003 Castellón. 